\title{
Uniparental isodisomy as a cause of recessive Mendelian disease: a diagnostic pitfall with a quick and easy solution in medium/large NGS analyses
}

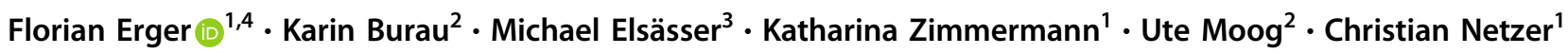

Received: 21 February 2018 / Revised: 10 April 2018 / Accepted: 22 May 2018 / Published online: 11 June 2018

(c) European Society of Human Genetics 2018

\begin{abstract}
Complete uniparental isodisomy (iUPD) — the presence of two identical chromosomes in an individual that originate from only a single parental homolog - is an underestimated cause of recessive Mendelian disease in humans. Correctly identifying iUPD in an index patient is of enormous consequence to correctly counseling the family/couple, as the recurrence risk for siblings is reduced from $25 \%$ to usually $<1 \%$. In medium/large-scale NGS analyses, we found that complete iUPD can be rapidly and straightforwardly inferred from a singleton dataset (index patient only) through a simple chromosome- and genotype-filtering step in $<1 \mathrm{~min}$. We discuss the opportunities of iUPD detection in medium/large-scale NGS analyses by example of a case of $C H R N G$-associated multiple pterygium syndrome due to complete maternal iUPD. Using computer simulations for several detection thresholds, we validate and estimate sensitivity, specificity, positive (PPV), and negative predictive values (NPV) of the proposed screening method for reliable detection of complete iUPD. When screening for complete iUPD, our models suggest that a $>85 \%$ proportion of homozygous calls on a single chromosome with $\geq 30$ sufficiently interspaced called variants results in a sensitivity of $97.9 \%$ and specificity of $99.7 \%$. The PPV is $95.1 \%$, the NPV 99.9\%. When this threshold is exceeded for a chromosome on which a patient harbors an apparently homozygous disease-associated variant, it should be sufficient cause to discuss iUPD as a plausible or probable mechanism of disease in the genetic analysis report, even when parental segregation has not (yet) been performed.
\end{abstract}

\section{Introduction}

In an individual with uniparental disomy (UPD), both alleles at a given locus within the diploid genome are inherited from only one parent. This usually occurs as a consequence of

Electronic supplementary material The online version of this article (https://doi.org/10.1038/s41431-018-0195-2) contains supplementary material, which is available to authorized users.

Florian Erger

florian.erger@uk-koeln.de

1 Institute of Human Genetics, University of Cologne, Cologne, Germany

2 Institute of Human Genetics, Heidelberg University, Heidelberg, Germany

3 Department of Prenatal Medicine, Heidelberg University Women's Hospital, Heidelberg, Germany

4 Center for Molecular Medicine, University of Cologne, Cologne, Germany errors in meiosis and subsequent zygotic rescue mechanisms [1]. Whereas in uniparental heterodisomy, the individual inherits both homologs from one parent, in uniparental isodisomy (iUPD), both alleles originate from a single-parental homologous chromosome and are therefore identical. The isodisomy can affect whole chromosomes ("complete iUPD") or parts of chromosomes ("segmental iUPD"). Complete UPD makes up $~ 90 \%$ of the reported cases in the online UPD database [2], although this figure may be biased due to the difficulty of detecting smaller segmental UPD. The incidence of all forms of UPD (including uniparental heterodisomy) has been estimated at around 1:3 500 births [1]. Besides imprinting-associated genetic disorders [3], iUPD can also lead to the occurrence of recessive Mendelian diseases, if one or more variants affecting function are present on the isodisomic allele. Many such patients have been reported to date [2]. Indeed, this pathomechanism may not be overly rare: among 73 patients with apparent homozygosity for diseaseassociated variants in an unselected cohort, Yang et al. [4] found four patients with complete iUPD $(\sim 5.5 \%)$ and one with segmental iUPD $(\sim 1.4 \%)$. 
Patients with complete iUPD are necessarily homozygous for all genetic variation on the affected chromosome. This loss of heterozygosity can, depending on analytical quality, be detected in routinely generated NGS data of a single individual using straightforward filtering techniques.

iUPD in the context of NGS remains a blind spot in most current diagnostic recommendations and has so far not been fully addressed in the current diagnostic NGS guidelines of the European Society of Human Genetics [5], or the relevant guidelines [6-10] of the American College of Medical Genetics.

We discuss the possible implications and opportunities regarding complete iUPD in diagnostic NGS by the presentation of a case of maternal isodisomy of chromosome 2 causing autosomal-recessive lethal-type multiple pterygium syndrome (OMIM \#253290). The diagnosis was identified in silico by targeted reanalysis of NGS variant calls without the use of dedicated UPD-detection tools and prior to marker analysis of parental samples.

\section{Materials and methods}

\section{Clinical report}

The index patient is the female fetus of a 35-year-old third gravida. The child's parents were of German origin, nonconsanguineous and had an unremarkable family history. In the 12th week of pregnancy (WoP), a strongly increased nuchal translucency of $6 \mathrm{~mm}$ was observed. During the further course of the pregnancy, prenatal ultrasound showed akinesia and contractures of the extremities (fixed and bent arms, closed fists, bent hips, and stretched legs), bilateral clubfeet, buckling of the thoracic spine, skin edema, suspected lung hypoplasia and polyhydramnios. Fetal growth corresponded to the 5th centile. NIPT and amniocentesis showed normal results $(46, \mathrm{XX})$. A severe form of arthrogryposis syndrome or fetal akinesia deformation sequence was suspected. After detailed gynecological and pediatric counseling, the parents opted for termination of pregnancy at $23+0$ WoP. Clinical and pathological examination of the fetus confirmed the prenatally observed abnormalities and additionally revealed facial dysmorphic features (hypertelorism, micrognathia, neck- and eyelid-edema) without further anomalies of the inner organs.

\section{Genetic testing}

Fetal DNA was extracted from umbilical cord tissue and enriched using a custom designed Agilent (Santa Clara, CA, USA) SureSelect gene panel containing 487 genes associated with neuromuscular Mendelian phenotypes ("NMD panel"). Sequencing was performed on an Illumina (San Diego, CA, USA) NextSeq500. The diagnostic pipeline is further described in Supplement.

Variants were classified according to the ACMG criteria [8].

\section{Segregation and diagnosis of iUPD}

Parental segregation was done by Sanger sequencing.

For reanalysis of the fetal NGS data, we filtered all called variants by chromosomal location and genotype and calculated the proportion of homozygous calls $(\mathrm{PoH})$ among all called variants on each chromosome.

We subsequently performed polymorphic tandem repeat marker analysis on fetal and parental DNA for the loci D2S309, D2S2188, D2S2193, D2S2309, D8S405, D9S172, D9S1784, D12S1590, D12S1604, D15S1018, and D17S1529 (Figures S1-S3).

\section{Validation of screening approach}

We performed a set of simulations using two whole-exome (WES) datasets without iUPD and our NMD gene panel dataset. WES sequence data were processed as described in Supplement. One WES-dataset originated from an individual without known parental consanguinity, the second from an individual with parental consanguinity (first-degree cousins). We took 1000 random samples of variant calls for each autosome under the following models:

(1) 20 randomly distributed variant calls

(2) 4 randomly distributed sets of 5 adjacent variant calls each

(3) 30 randomly distributed variant calls

(4) 6 randomly distributed sets of 5 adjacent variant calls each

We analysed $\mathrm{PoH}$ in each random sampling and calculated sensitivity, specificity, positive (PPV), and negative predictive values (NPV) with regard to iUPD-detection for each of the tested models.

\section{Data accession}

Relevant patient variant and phenotype data were submitted to https://www.lovd.nl/CHRNG (patient ID 163650), as well as to the NCBI's ClinVar database (variation ID 487641).

\section{Results}

Among 13 genes for the more frequent types of fetal akinesia syndrome, only a single-homozygous variant, c. $1210 \mathrm{C}>\mathrm{T} \quad\left(\mathrm{p} .\left(\mathrm{Gln} 404^{*}\right)\right)$ in the $C H R N G$ gene 
Table 1 Performance of a cutoff of $\geq 85 \%$ homozygous calls per chromosome by extrapolation from several thousand simulations on real-life WES and gene panel data

\begin{tabular}{|c|c|c|c|c|c|}
\hline Model & $\begin{array}{l}\text { Tested autosomes } \\
(n)\end{array}$ & $\begin{array}{l}\text { False positive } \\
(n)\end{array}$ & $\begin{array}{l}\text { False negative } \\
(n)\end{array}$ & Sensitivity & Specificity \\
\hline 20 variants NCS & 22 & 2 & 0 & - & $99.9 \%$ \\
\hline $4 \times 5$ variants $\mathrm{NCS}$ & 22 & 75 & 0 & - & $99.7 \%$ \\
\hline 30 variants NCS & 22 & 0 & 0 & - & $100 \%$ \\
\hline $6 \times 5$ variants $\mathrm{NCS}$ & 22 & 10 & 0 & - & $99.9 \%$ \\
\hline 20 variants $\mathrm{CS}$ & 22 & 21 & 0 & - & $99.9 \%$ \\
\hline $4 \times 5$ variants $C S$ & 22 & 296 & 0 & - & $98.7 \%$ \\
\hline 30 variants $\mathrm{CS}$ & 22 & 0 & 0 & - & $100 \%$ \\
\hline $6 \times 5$ variants $C S$ & 22 & 59 & 0 & - & $99.7 \%$ \\
\hline $\begin{array}{l}20 \text { variants UPD } \\
\text { panel }\end{array}$ & 17 & 2 & 2 & $99.8 \%$ & $99.9 \%$ \\
\hline $\begin{array}{l}4 \times 5 \text { variants UPD } \\
\text { panel }\end{array}$ & 17 & 59 & 11 & $98.9 \%$ & $99.6 \%$ \\
\hline $\begin{array}{l}30 \text { variants UPD } \\
\text { panel }\end{array}$ & 14 & 0 & 0 & $100 \%$ & $100 \%$ \\
\hline $\begin{array}{l}6 \times 5 \text { variants } U P D \\
\text { panel }\end{array}$ & 14 & 1 & 21 & $97.9 \%$ & $99.9 \%$ \\
\hline
\end{tabular}

We performed 1000 simulations per autosome. NCS: no parental consanguinity exome; CS: parental consanguinity exome; UPD panel: gene panel data from individual with iUPD of chromosome 2
(NM_005199.4, OMIM *100730) remained after our routine filtering steps. This novel variant fulfilled the ACMG criteria PVS1, PM2, and PP3, and was classified as "Pathogenic", establishing the diagnosis of lethal-type multiple pterygium syndrome (OMIM \#253290) in the index patient.

Parental segregation analysis confirmed the c.1210C $>\mathrm{T}$ $C H R N G$ variant in the maternal sample in a heterozygous state. It was not detected in the paternal sample. NGS-based copy number variation (CNV) detection did not suggest any $\mathrm{CNV}$ in the patient sample.

The proportion of homozygous calls $(\mathrm{PoH})$ in the fetal NGS-sample was highly increased on chromosome 2, where $C H R N G$ is located. Among all 96 called variants on chromosome 2 (Figure S4), 95\% were homozygous. The remaining heterozygous calls were classified as sequencing or alignment artefacts in GC-rich or repetitive regions upon individual inspection. This in silico filtering step, demonstrating complete maternal iUPD with very high confidence, was completed within $<1 \mathrm{~min}$. Maternal iUPD of chromosome 2 was subsequently further confirmed through marker analysis of the fetal and parental samples, whereby non-paternity could also be excluded (Figures S1-S3).

With a threshold of $\geq 85 \% \mathrm{PoH}$, our simulations showed a sensitivity for complete iUPD detection of $98.9 \%$ for $\geq 20$ variant calls and $97.9 \%$ for $\geq 30$ variant calls. Specificity was $98.7 \%$ for $\geq 20$ variant calls and $99.7 \%$ for $\geq 30$ variant calls (Table 1; Figures S5-S12). Estimating a frequency of iUPD of 5\% among patients with homozygous diseaseassociated variants [4], we calculate a PPV of $95.1 \%$ and an
NPV of $99.9 \%$ for a threshold of $\geq 30$ variant calls and $\mathrm{PoH}$ $\geq 85 \%$.

\section{Discussion}

With as few as 30 variant calls in 6 or more clusters along the length of a single chromosome (the average of the variants' allele frequencies should be $\sim 50 \%$ in an unbiased and unfiltered selection) and a $\mathrm{PoH}$ of $\geq 85 \%$, we show that complete iUPD can be confidently inferred from a singleNGS dataset. With this high threshold, even homozygosity by descent in the offspring of first cousins did not lead to a high rate of false-positives; although the false-positive rate may be increased in populations with higher levels of (multigenerational) consanguinity, especially for small chromosomes. Since genomic regions in close proximity may be in linkage disequilibrium, the 6 or more variant clusters should be spaced $\geq 1 \mathrm{Mb}$ apart to prevent oversampling from a single-haplotype block. One should keep in mind that a low-analytic quality may introduce a larger number of erroneous heterozygous variant calls, thereby depressing the measured $\mathrm{PoH}$. We recommend applying this filtering step to high-quality variant calls only.

Although the parental segregation of a homozygous variant is routinely recommended, DNA of both parents is not always available or made available for follow-up testing. One previous study reported a "segregation compliance" for homozygous variants of 25-30\% [11], although this is higher-roughly $50-60 \%$-in the authors' personal experience. 
The major advantages of the filtering/screening method described herein are its simplicity and speed. It can be performed by the genetic specialist directly at the time of primary analysis when a homozygous variant affecting function is detected. No additional bioinformatic tools need to be integrated into the established pipelines. We recognize that small to medium-sized segmental iUPD will most probably escape detection by this method. Uniparental heterodisomy cannot be distinguished without parental controls, although in the setting of recessive disease this would require the parent-of-origin to be affected as well. Additionally, detection of iUPD in trio-analyses can be achieved by directly comparing the parent and child genotypes $[12,13]$.

iUPD has previously been detected in NGS data using bioinformatic tools that operate essentially by detecting runs of homozygosity $[14,15]$ or by genotype comparison in trio-sequencing data [13]. These tools are more likely to also detect smaller segmental iUPD but require a more complex setup and bioinformatic know-how and are not, to the authors' knowledge, widely used in the diagnostic routine. Tools intended to be used on WES data [15] may not fare as well when used on gene panel data.

Our aim is not to present a complex, be-all-end-all method for in silico detection of all forms of iUPD. Rather, we propose a screening method to identify obvious cases of complete iUPD (which likely make up a sizeable majority of clinically relevant iUPD cases $[2,4]$ ) during medium to large NGS analyses at the cost of less than a minute of the analyst's time.

Routine screening for complete iUPD will only influence the final interpretation of the molecular genetic findings in a small, but significant subset of cases (possibly $>5 \%$ of cases with homozygous disease-associated variants [4]). However, the significance for the parents and/or family of the affected individual is enormous. For one, the recurrence risk for siblings drops from $25 \%$ to usually $<1 \%$, depending on the carrier frequency of the respective disorder. In the sizeable percentage of cases that are not followed up with segregation testing, this may impact future family planning. In cases where invasive prenatal testing might otherwise have been performed without prior segregation testing, such unnecessary prenatal risks can be avoided.

Given the simplicity of detecting complete iUPD in medium to large NGS-based analyses (gene panels, WES, WGS), we propose that complete iUPD be routinely and immediately excluded upon identification of a homozygous disease-associated variant in any patient sample that is analysed using one of these tests. To our knowledge, despite its ease, this is not currently done in the large majority of accredited genetic diagnostic laboratories, thereby leaving iUPD discovery to only those cases where segregation analyses can be performed.

Acknowledgements The authors are grateful to the patient's family for their participation in this project.

\section{Compliance with ethical standards}

Conflict of Interest The authors declare that they have no conflict of interest.

\section{References}

1. Robinson WP. Mechanisms leading to uniparental disomy and their clinical consequences. Bioessay. 2000;22:452-9.

2. Liehr T. Cases with uniparental disomy. 2017. Accessed 15 Dec 2017.

3. Mackay DJG, Temple IK. Human imprinting disorders: principles, practice, problems and progress. Eur J Med Genet. 2017;60: 618-26.

4. Yang Y, Muzny DM, Xia F, et al. Molecular findings among patients referred for clinical whole-exome sequencing. JAMA. 2014;312:1870-9.

5. Matthijs G, Souche E, Alders M, et al. Guidelines for diagnostic next-generation sequencing. Eur J Human Genet. 2016;24:2-5.

6. Shaffer LG, Agan N, Goldberg JD, Ledbetter DH, Longshore JW, Cassidy SB. American College of Medical Genetics statement of diagnostic testing for uniparental disomy. Genet Med. 2001;3:206-11.

7. Richards CS, Bale S, Bellissimo DB, et al. ACMG recommendations for standards for interpretation and reporting of sequence variations: revisions 2007. Genet Med. 2008;10:294-300.

8. Richards S, Aziz N, Bale S, et al. Standards and guidelines for the interpretation of sequence variants: a joint consensus recommendation of the American College of Medical Genetics and Genomics and the Association for Molecular Pathology. Genet Med. 2015;17:405-24.

9. Rehm HL, Bale SJ, Bayrak-Toydemir P, et al. ACMG clinical laboratory standards for next-generation sequencing. Genet Med. 2013;15:733-47.

10. Maddalena A, Bale S, Das S, Grody W, Richards S, Committee ALQA. Technical standards and guidelines: molecular genetic testing for ultra-rare disorders. Genet Med. 2005;7:571-83.

11. Landsverk ML, Douglas GV, Tang S, et al. Diagnostic approaches to apparent homozygosity. Genet Med. 2012;14:877-82.

12. Schroeder C, Sturm M, Dufke A, et al. UPDtool: a tool for detection of iso- and heterodisomy in parent-child trios using SNP microarrays. Bioinformatics. 2013;29:1562-4.

13. King DA, Fitzgerald TW, Miller R, et al. A novel method for detecting uniparental disomy from trio genotypes identifies a significant excess in children with developmental disorders. Genome Res. 2014;24:673-87.

14. Bis DM, Schule R, Reichbauer J, et al. Uniparental disomy determined by whole-exome sequencing in a spectrum of rare motoneuron diseases and ataxias. Mol Genet Genom Med. 2017;5:280-6.

15. Magi A, Tattini L, Palombo F, et al. H3M2: detection of runs of homozygosity from whole-exome sequencing data. Bioinformatics. 2014;30:2852-9. 